\title{
Block-Chain Technology -Security, Platforms
}

\author{
Ammbika V M, D.S Rao
}

\begin{abstract}
The current paper is focused on its security issues with its prospective applications for educational applications and explored how blockchain Technology can be used to solve some education problems.Blockchain Technology can be integrated into multiple areas like cryptocurrencies,smart contract ,Bank,Blockcahin with video games, finance,judiciary, commerce and IOT.Blockchain Technology has already changed peoples lifestyle in many areas due to its great influence.

In this paper the Three types of Blockchain engineering 1.0,2.0,3.0 with its protocol stack layer working, its advantages and applications are checked.Along with different platforms on which Blokchain is running with its features is highlighted and evolution phases in Blockchain.This paper aim is to test amenities of security,related to the Blockchain and need of innovation of Blockchain.
\end{abstract}

Index Terms: Blockchain-Trust, Immutable, security ,services, EducationTechnology .

\section{INTRODUCTION}

A block chain is essentiallya block of chain with growing list of records referred to as blocks that are joined with cryptography. Each block chain contains a hash(i.e a special category of hash perform that has bound properties that are utilized in cryptography)of a previous block, a timestamp(keeping track of the creation and modification time of a document. Security i.e. nobody -not even the owners of the document ought to be able to modify it once it's been recorded only if the timestampers integrity is rarely compromised.Block chain was invented by santoshi Nakamoto in 2008 and it is implemented in 2009 to function the general public dealings ledger of the cryptocurrency (digital asset) bitcoin (cryptocurrency) a type of electronic money .Blockchain Technology is decentralized(i.e peer to peer).It consists a world network of computers, uses blockchain technology to put together and manage the information that records each bitcoin dealings and managed by its network.It has been thought of as a part of the reality technological revolution since the invention of stream engine ,electricity Technology (chung and Kim 2016, Schwab 2017). Fig Below shows traditional and Blockchain N/w

Revised Manuscript Received on 10, September 2019.

* Correspondence Author

Miss Ammbika Vamanrao Mittapally, Computer Science And Enggineering, Doctoral Research Scholar -CSE,Koneeru Lakshmaiah Education Foundation(KLEF) ,Vaddeswaram, Guntur, Andhra Pradesh-522502, India.

D.S.Rao,Associate Professor,Computer Scienceand Enggineering,Associate Dean $(\mathrm{P} \& \mathrm{D})$, ,Koneeru Lakshmaiah Education Foundation(KLEF) ,Vaddeswaram,Guntur District, Andhra Pradesh-522502, India.

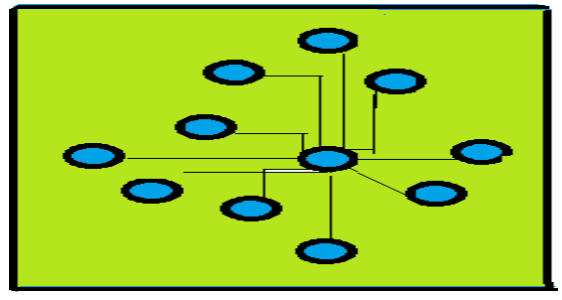

Fig 1:Traditional Centralized Block chain Network

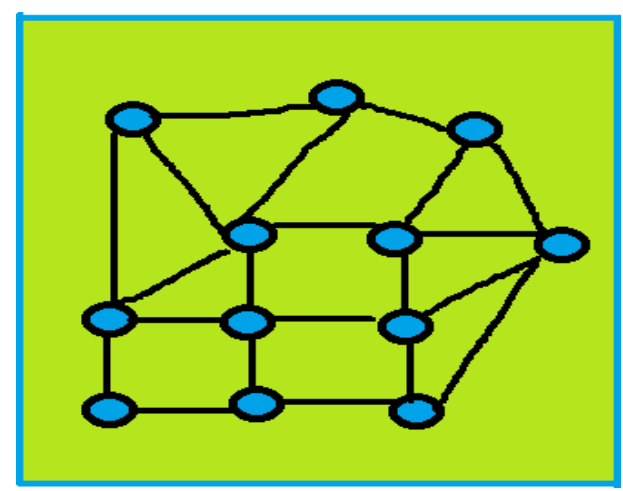

Fig 2: Blockchain Network for Peer -to-Peer Technology

In this paper, we will have a fast study regarding what's blockchain in section 2 , then we will discuss regarding security problems associated with blockchain technology in section 3 , at the end,we shall mention the services provided by blockchain technology section 4 , explored however blockchain technology are often employed in education system, the paper is all over in section [5].

\section{DIFFERENT PHASES AND GENERATIONS OF BLOCKCHAIN TECHNOLOGY}

For convenience and relying upon new inventions ,the new quite activity alongside its existing activities within the Blockchain generations categorized into 3 stages.

Blockchain one.0, Blockchain a pair of.0, Blockchain three.0.[1,5,6]

Blockchain one.0-Digital currency : which is expounded to cryptocurrencies in applications associated to money, like exchange currency, transfer currency, digital payments systems.It works for Peer -to-Peer cash and Peer-to-Peer payments[1,5,6].

Blockchain a pair of.0-Digital Economic: which is related to economic market applications victimization the Blockchain that are intensive than easy money transactions, stock, exchange, bonds, titles, smart property, sensible contracts, loans and mortgages[3]. 
Blockchain three.0-Digital Application: for society which might be used on the far side currency, finance and markets-specifically in governments sectors, health, science, literacy, culture and art ,identity, education, public product, communication[8].

Block chain Technology stack additionally known as as 3 Tier Blockchain Technology one.0:

Bitcoin-Virtual currency, stack of protocols,Blockchain Technology are 3 layers that are still in development part

.Communication among completely different layers in Blockchain Technology is Full duplex i.e 2 approach communication i.e causation and receiving of knowledge takes place in each direction. Firstly, information is distributed from Blockchain Technology to stack of protocols(which consists of some set of rules and rules for communication i.e criteria)it works with communication protocols like TCP/IP-Transport protocols-Middle layer.Middle layer will 2 approach communication with Bitcoin -Virtual Currency for transmission and reception of knowledge blocks[8]

The Bitcoin may be employed in 3 alternative ways.

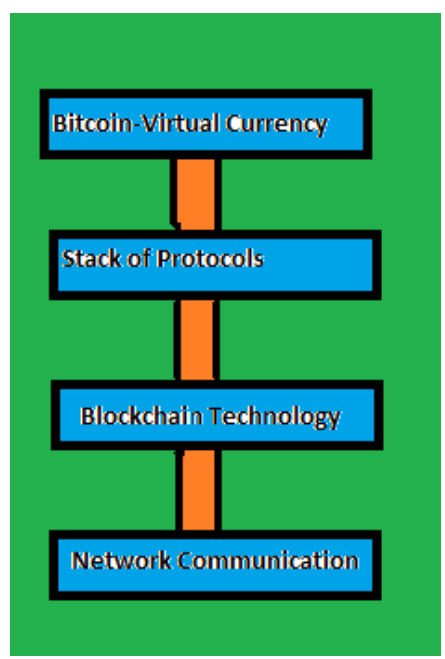

Fig 3: Communication among different layers in Blockchain Technology

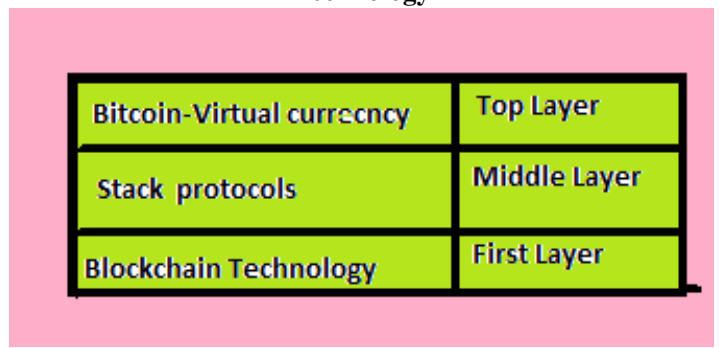

Fig 4: Blockchain Technology Stack Model in Blockchain 1.0 Bitcoin is favorite and really wide used crypto currencies.

Different layers in Bitcoin stack-Blockchain Technology. A:Blockchain works on the idea of Peer-to-peer Technology that include clear ledger the info file that is accessible on all Blocknode in network, altered by miners, administered by all block nodes and handled by nobody[6].

B:The second layer -middle tier of the stack protocol - which include software package with some rules and rules used for cash transfer and information is updated on Blockchain
ledger.It include TCP/IP-Transport layer for transmission and reception of knowledge on net victimization google chrome, Netscape etc[8].

The Third Tier-top layer -digi money that is Bitcoin virtual currency denoted as BTC or Btc- for group action and exchanges[9].It works victimization the hash technology,digital signature, public key,Peer-to-Peer, Proof-of-work-which enable users to form Bitcoins. Alternative Bitcoins are Ripple, Litecoin, ,Dogecoin, NXT Peer-coin, alt_currencies[12].

\section{Advantages of Biticon; \\ Bitcoin:}

a:Name itself indicates that it works bit by bit[4].

b: Allows on-line purchase and on-line group action.

c: Works a lot of versatile than master card.

d: Price grows at regular intervals.

e: Permits versatile non-reversible group action.

$\mathrm{f}$ : It's like digital money group action.

\begin{tabular}{|l|l|}
\hline First & Bitcoin-Blockchain Technology Platform \\
\hline Second & Protocol-software which exectues for tranfer assests on Blockchain \\
\hline Third & Digital Currency \\
\hline
\end{tabular}

Fig 5: Bitcoin uses.

In short any cryptocurrency structure/Model include:

A: Blockchain

B:Protocol

C:Currency

Mostly coin include currency \& protocol with their own

Blockchain or they execute victimization Bitcoin

Blockchain[9].

For example, it additionally include 3 Tier design.

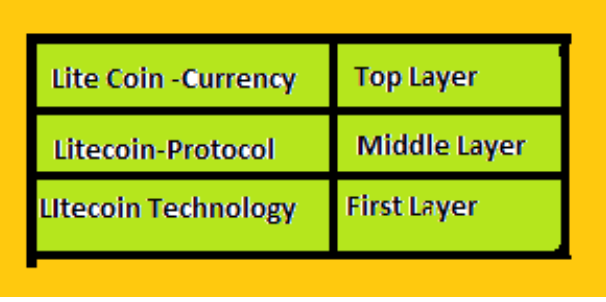

Fig 6: Litecoin Technology with stack Model

\section{Blackchain a pair of 0. -digital Economy:}

which incorporates Bitcoin a pair of.0 that consists of -Protocols, sensible property,sensible contracts, Dapps(decentralized Application)[7]

,Dao (Decentralized Autonomous organizations)

DACs-Decentralized autonomous firms.

Stack protocol-consist of communications protocol, SMTP, FTP of Blockchain a pair of.0 that is employed for linking, causation email, transfer of file on web victimization Napster a pair of.0, Facebook, WebBrowser3.0 


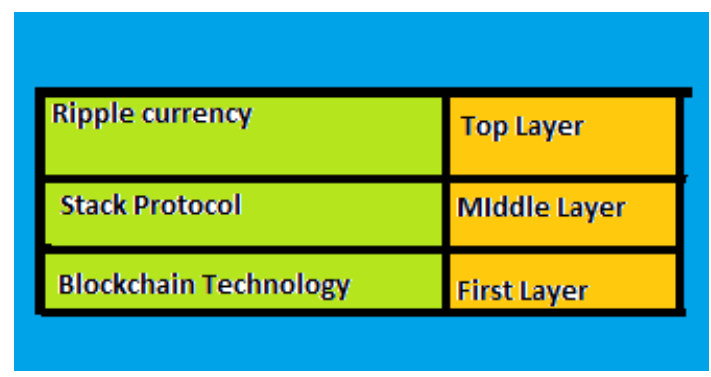

Fig 7: Ripple Technology with stack Model

This concept came into existence twenty two years past ,but recently it's uncombable with new rising technology platform.New Technology concerned in Blockchain a pair of.0 is sensible contracts[3]. they're principally software package applications that mechanically run the terms of contract.In sensible contract once bound condition is glad by the concerned parties during a written agreement agreement ,then payments may be created as per the contract that is all clear[3].

Ethereum: in style platform that runs for sensible contracts[4].

\section{Advantages of Ripple:}

A: it's open supply with distributed agreement ledger[4].

B: Uses RippleNet-send cash globally

C: Speed:High speed on demand settlement.

D: value:Low operational cost.

E: Certainty: Real-Time.

\section{Decentralized Application(Daap):}

\section{Features:}

A: Application- open supply.

B: Operate severally i.e no body dominant the bulk of its token.

C: info File:Stored Public.

D: Operations on File:Public

E: Topology:Peer-to-Peer.

\section{Applications:}

Mostly application should manufacture tokens victimization commonplace algorithmic rule or set of criteria[10].These tokens are employed by several application.

Market Response:

Application adapts protocol in co-ordinance with its projected enhancements \& market response[12].All changes are determined upon the agreement of its users.

Projects employed in Dapps: Lazoz, Twister,Gems, Storj, OpenBazzar[12].

Blockchain 3.0-Digital Application-Digital money. Mostly used for sensible cities as well as at numerous sectors like sensible governance, sensible quality, sensible living, the sensible use of natural resources, sensible on-line, sensible voters, sensible economy[9]. Blockchain Technology operates each internally and outwardly considering quality and amount characteristics. Blockchain 3.0 is wide used, in

needed list for world wide and operates at minimum worth [10].

\section{Blockchain Learning:}
A: Peer-to-Peer Learning
B: Learning is financial gain supply.
C: Moocs: large on-line Course detached world wide singly.
D: Peer-to-Peer learning contracts.

\section{Blockchain Health:}

Popular idea associated with digital health quality.Blockchain Health application may be used for Health connected applications[13].

\section{Advantages:}

A: Health information may be recognized however information is unbroken non-public[13].

Platform: Blockchain Technology works on numerous platform 1: Ethereum 2: Hyperledger, coin or little contracts bound, it is based on various algorithmic, developing tools and programming languages.

1: Ethereum: This supports decentralized platform that runs good contracts: applications that run precisely as programmed with no risk of down time,(fraud or third party interference). Ethereum permits developers to form markets, store registries of debts or guarantees. On a Blockchain anyone will setup a node that replicates the mandatory knowledge for all nodes to achieve an argument $\&$ be salaried by users and app developers[3].It allows user knowledge to stay non-public and app to be decentralized just like the web was expect to work.

2: Hyperledger: Hyperledger is Associate in Nursing open supply cooperative effort created to advance cross-industry blockchain technologies[27]. It's a worldwide collaboration and it is hosted by The Linux Foundation, together with leaders in finance, banking, IoT[27 ], supply chain, manufacturing and technology.

Table 1:Comparison of Ethereum and Hyperledger

\begin{tabular}{|l|l|l|l|}
\hline Sr no & characteristic s & Ethereum & Hperledger \\
\hline $\mathbf{1}$ & $\begin{array}{l}\text { Permission } \\
\text { Restrictions }\end{array}$ & $\begin{array}{l}\text { Permission less } \\
\text { access } \\
\text { to data }\end{array}$ & Permissioned \\
\hline $\mathbf{3}$ & Consensus & Proof-of-work & PBFT \\
\hline $\mathbf{4}$ & Scalability & $\begin{array}{l}\text { High Node } \\
\text {-scalibility,Low } \\
\text { performance-scal } \\
\text { ability }\end{array}$ & $\begin{array}{l}\text { Low } \\
\text { node-scalability, } \\
\text { High } \\
\text { performance-scal } \\
\text { ability }\end{array}$ \\
\hline $\mathbf{5}$ & $\begin{array}{l}\text { Centralized } \\
\text { regulation(gov } \\
\text { erance } *)\end{array}$ & $\begin{array}{l}\text { Medium core } \\
\text { developer group,but } \\
\text { EIP } \\
\text { process }\end{array}$ & $\begin{array}{l}\text { Low,open-gevern } \\
\text { ance model based on } \\
\text { Linux Model }\end{array}$ \\
\hline $\mathbf{6}$ & $\begin{array}{l}\text { Anonymity } \\
\text { Pseudonymity,no } \\
\text { encryption of } \\
\text { transaction data }\end{array}$ & $\begin{array}{l}\text { Pseudonymity.en } \\
\text { cryption of } \\
\text { transaction data }\end{array}$ \\
\hline $\mathbf{7}$ & $\begin{array}{l}\text { Native } \\
\text { currency }\end{array}$ & $\begin{array}{l}\text { Yes,ether } \\
\text { No }\end{array}$ \\
\hline
\end{tabular}




\begin{tabular}{|l|l|l|l|}
\hline 8 & scripting & High & High \\
& possibility,Turin \\
g-complete virtual & possibility,Turin \\
machine,high-lev el & g-complete scripting \\
& of chaincode,high-1 \\
& & language & evel \\
& & Gopport(solidity) & Go-language. \\
\hline
\end{tabular}

\section{EVOLUTION PHASES IN BLOCKCHAIN TECHNOLOGY:}

Recently Blockchain is categorized in 3 phases relying upon its economic criteria, political price, humanitarian and edges of system Bitcoin, scientific-(inventions)filed[2] .In economic and for political edges ,the coordination, database, irrevocability of transactions victimization blockchain technology are options house that might be as basic for any progress in society because the Magna Charta[6]. In this case, the blockchain will function the general public record repository for societies, as well as the register of all documents, events, actions, activities, establish and assess of it[7].

In this system, all property would become sensible property[2] .This becomes the identity of encryption every quality in blockchain with its distinctive identity.so as quality are copied controlled by, sold on the blockchain.This indicated that every one daily victimization assets like automotive, mobile, house land, shapes and alternative digital assets may be registered group action may be done on Blockchain[7].For example, we will say that world ever-changing potential of the Blockchain for its use in distinctive registering intellectual property(IP)[2].

Now a days, the leading digital art trade provides digital services for in camera registering the small print of any digital gadgets or digital asset(like image ,file, anamnesis, hardware, CPU, camera, watch, software,Xerox machine) on the blockchain[1].The Blockchain might replaces all out there Intellectual property(IP)management systems. Mostly, it works victimization commonplace algorithmic rule that is run on a particular file or any file that is to be compressed into a brief sixty four character secure hash that isn't calculated backward[2].The hash values are added during a Blockchain group action, for timestamp price the Proof-of that digital quality price at that individual movement[2] .

The hash price may be verified from the previous file(which is hold on in camera on the homeowners pc, not on the Blockchain )providing that maintaining the originality of the contents gift in it[7].

\section{SECURITY ISSUES}

Though the security issues of Blockchain innovation enables us to settle twofold spended problem, including its administrations. Blockchain innovation can be utilized in instruction framework to take care of a portion of its issues which is an essential point $[15,16,17]$.

\section{The Blochchain Technology comprises five primary charactersitics:}

\section{1: Blockchain Decentralized:}

The primary component of blockchain innovation is it doesn't rely upon any third party for exchange i.e shared.The

information can be recorded, stored, updated in distributively[15].

2 : Immutable:Any records are composed and put away for all time with no progressions except if anyone assumes responsibility for it[16].

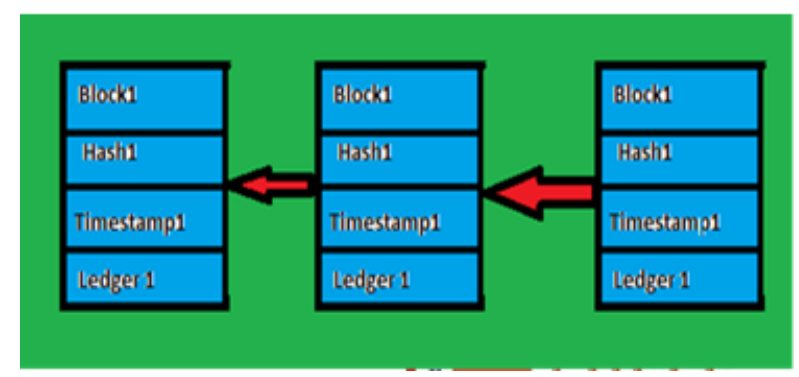

Fig 8. Attributes of Blockchain

3: Transparent: Blockchain can be tructed as a result of its straightforward nature and each block can be entered into it[17].

4: Persistency: Blocks with invalid transactions can be recognized, approved rapidly and invalid transactions are not conceded by genuine miners [15]. It is about difficult to erase or rollback transactions, once they are incorporated into the Blockchain[17].

5: Trust: Block chain Technology is dependable and it offers secure activities like payments, or issue of certificates, record keeping. Since the records are shared among a network of sealed nodes[15].

6: Timestamp: It is time of block created and measures of time spend in doing transactions [16].

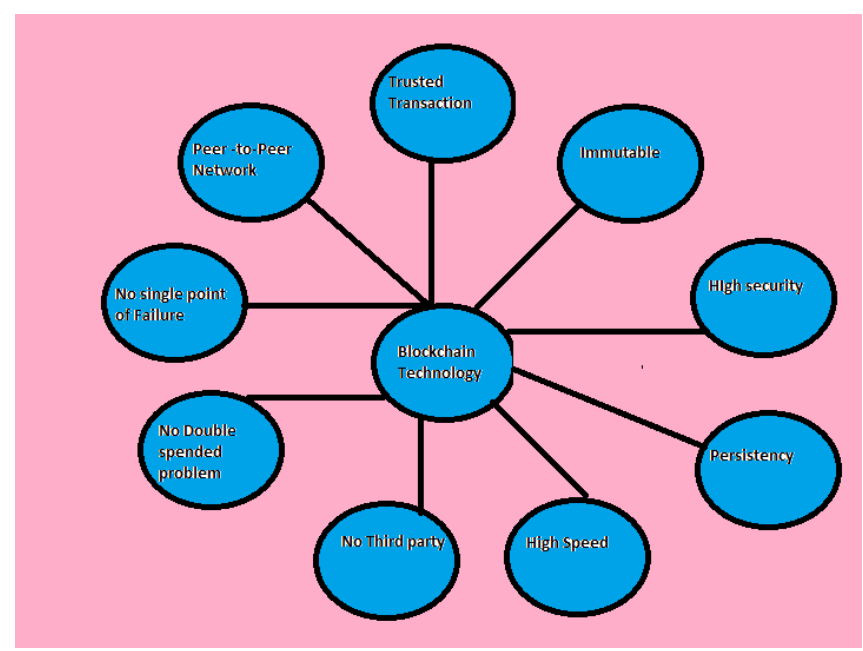

Fig 9: Characteristics of Blockchain Technology.

\section{Security issues Related to blockchain innovation}

1: DNS ATTACKS: Sending peers wrong data [13]. 2: Mempool Attacks: Flooding new blocks with transactions [14]. 
3: Selfish Mining: Miners endeavoring to expand their prizes by keeping blocks private [15].

4: $51 \%$ attack: Someone achieves majority share in a system and misuses it $[11,13]$.

5: Forks:That can be brought about by sybil nodes or selfish mining[15].

6: Double spending attacks: Creating two transactions from the equivalent upsent transaction $[11,3,12]$..

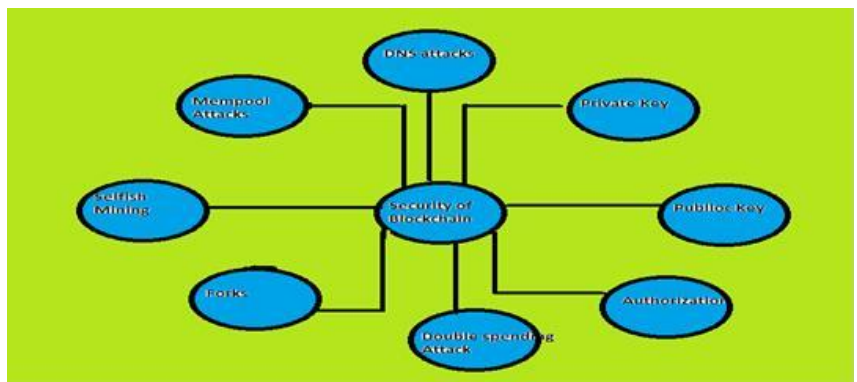

Fig 10 : Securities issues of Blockchain Technology.

Why Blockchain innovation need to characterize security.

There is no denying that Blockchain is every now and again depicted as an at last secure arrangement in numerous industries, starting from money transaction, ending with advertising automation. When the state of Michigan introduced a bill to punish controlling blockchain information, they made the primary legitimate endeavor to ensuring information put away and transmitted through conveyed frameworks. This legislation didn't simply show up out of nowhere thinking about the cryptographic money, it just appears to be consistent to take activities to lawfully punish these attempts. This prompts a question: If the innovation should be ensured legally, what do we mean by sparing that blockchain is secure?

\section{A few reasons why blockchain network needs a superior security.}

Reason \#1: Decentralized systems won't be as secure as they appear.

Robust case in favor of blockchain technology is that with copies of blockchain being unbroken on a cosmopolitan $n / w$, there is nobody week purpose for hackers to focus on such, theory would possibly work for smaller systems that don't suppose mining the maximum amount. If everybody will be part of the $\mathrm{n} / \mathrm{w}$, how will we check that the safety isn't in risk [2].

The answer here is often a permissioned system, where potential members are verified [3]. This but creates a lot of queries than answers, who is to blame of determining whether the participants area unit secure? Who deserves to possess that sort of power?

\section{Reason \#2: Attacks on market places}

On December 2017, nice hash, one of the biggest Bitcoin market places got hacked, resulting in million in losses [6].
It raises a crucial issue. If blockchain operations are safety. Technically it was not the fault of the technology since it had been not Bitcoins vulnerability that created the attack potential. The weakness within the market place itself price a great deal to Bitcoins investors [7].

As blockchain gets much popular, so do third party marketplaces and distributors however not all of them area unit are safe [8]. They might not be as secure as users or investors would really like to think-\&the Nice Hash attack simply evidenced it [8].

\section{Reason \#3 : Smart contracts will malfunction.}

If enforced right, smart contracts will really answer several of the present problems in current selling, sales, investment, and development method [18]. However, if the contract incorporates a tiny bug, it will place the signed parties in risk. In 2016, it happened with associate degree Ethereum contract, worth \$ eighty million fortuitously; Ethereum community was ready to resolve the problem by making it look as nothing is going on [18]. Developers have written a replacement version of history wherever the attack was deleted.As nothing ever happened. However effective Ethereums community live was

,its none the less arguable. If any transaction are often written this manner, couldn't it negatively impact the worth of cryptocurrency?[18] Such fast -and-dirty fixes would possibly solve a 1 time crisis however on a permanent resolution ,we need something a lot of clear and reliable[18].

\section{Reason 4\# : "51" attack.}

Once the blockchain transaction is created ,miners who hold at least $50 \%$ of computing power got to verify the attack[11].If they did, the transaction will pass. Now imagine matters within which one person or a bunch of individuals may concentrate in their hands $51 \%$ of mining power[13]. This owner becomes the sport charger, acquiring prospects to pass transactions therewith same coin doubly, or as repeatedly as needed[11,13]. It breaks all the rules. which will never happen ,one may assume.In 2014, Ghash.io pool got near to dominant $51 \%$ of mining power that caused several miners to go away the pool and worry for the integrity of blockchain[11,13].51 systems attack appearance unrealistic as a result of cryptocurrency house owners learnt to right away notice abnormal additions to hash rate and neutralize them by adding a lot of power[11,13].

Zen cash has recently suffered the $51 \%$ attack \& paid a great transaction for that $[11,13]$.

\section{Reason 5\# : The size and complexness of a blockchain network.}

Tiny systems perpetually face nice possibilities of $51 \%$ attack. Since miners want less resources to execute it. Bigger $n / w$ area unit safer in this regard however insecure in several others [14].

Let's take Bitcoin, for instance Growing quick, the $n / w$ becomes significantly larger everyday \& not every miner is totally tuned in to potential threats \& technology weakness [14].By sharing scientific discipline keys-a mistake in skilled miners. Often make, they place

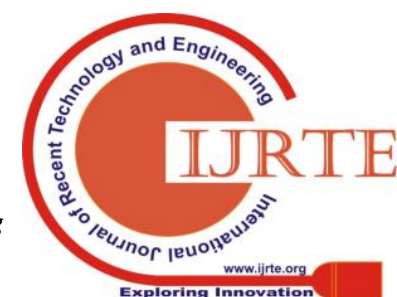


themselves at risk-\&compromise the complete system [21].

Running an enormous distributed system needs powerful n/w. If Network grows too quick, infrastructure will fail to support it[38].

In short, there area unit huge security problems that none the less got to be tackled [14]. Yet other is not any denial that blockchain is about as clear \& reliable as technology ever gets. We can either defend our n/w's from being attacked by dominant hash power \& cooperating with evidenced, third-party distributors or become explanation for associate degree attack.

Despite of nice security problems, it conjointly has several various services, which can be enclosed in blockchain.We enumerate service provided by blockchain technology in education System.

Blockchain as a service (Baas):using this customers can create cloud-based solutions, they can host \& use their own blockchain apps, smart contracts \& functions on the blockchain whereas the cloud-bases service supplier manages all the mandatory tasks and activities to stay the infrastructure agile.

\section{1: Intelligent Transportation systems (ITSC):}

Blockchain are often used to determine a secured ,trusted \& decentralized system[22] . It's system, creating higher usage of its infrastructure and resources, especially effective for crowdsourcing technology.

\section{2: Trust-Free system:}

It's trust free systems supported blockchain technology promise to revolutionize interactions between peers that need high degrees of trust. Usually expedited by third party suppliers. Peer-to-Peer platforms for resource sharing represent often discusses field of application for "trust-free" blockchain technology. However, It is the trust between peers which plays an important role in nearly all sharing economy interactions [25].

\section{3: Distributed Ledger Technology (DLT):}

It could be a info design that permits the keeping $\&$ sharing of records in distributed method, while guaranteeing its integrity through the employment of consensus-based validation protocols signatures[23]. In principle, DLT has the potential to scale back prices \& increase the potency of securities settlement, the ultimate step of each security transaction.

\section{Blockchain Technology are often used to unravel some education systems drawback.}

The blockchain Technology is deeply interconnected with totally different foundations of society, education, culture, charity \& even identity, government, economy [23]. In fourth coming back years Blockchain is associate degree intelligent digital mesh, which can connect individuals, devices, content \& services [24].

Education is domain wherever several national, international institutional analysis for integration the blockchain technology in attention-grabbing \& innovative applications such as: proof-of-work[3], proof-of-stake[4], proof-of-learning[5], management of credentials \& transcripts, management of student records[7], management of name $\&$ payments.

Supported the blockchain property of serving as a decentralized, trust-free[6], immutable[7] store of every type of data or assets, a group of pioneering universities began to experiment \& issue cryptographically signed, verifiable certificates on blockchain, which students will access or share with employers[8].

\section{CONCLUSION}

The simplest (\& worst) half regarding blockchain security is its constant growth. As technology develops, it becomes a lot of enticing for hackers. The strikes rise higher \& there is nothing we can do it.There continuously area unit growing to be new attack \& new ways that to prevent them. But as technologies, blockchain extremely is the definition of security.

Blockchain ends up in autonomous \& free information handling, to support communication \& education. Discipline is a fresh blockchain platform for comes within the instructional \& recruiting spheres, aiming to give the transparency of labor confidentiality \& reliability of data else by system participants.

Blockchain Technology in education system are often used since its teaching \& learning initiatives.

\section{REFERENCES}

1. KyleBurgessandJoeColangelo.The Promise of Bitcoinand the Blockchain.Consumers'Research,2015

2. MichaelCrosby,Nachiappan,PradhanPattanayak,SanjeevVerma, andVi gneshKalyanaraman.Blockchaintechnology:Beyondbitcoin,http://scet. berkeley.edu/wpcontent/uploads/blockchainpaper.pdf,2015.

3. AnastasiaBarinovaandSergeyZapechnikov.Onthetechniquesandtoolsf orprivacy-preservingsmartcontracts.Bezopasnostinformacionnyhtehn ology,2017(2),2017.

4. YeGuoandChenLiang.Blockchainapplicationandoutlookinthebanking industry.Financial Innovation,2(1):24,20

5. TylerMoore.Thepromiseandperilsofdigitalcurrencies.InternationalJour nalofCriticalInfrastructureProtection,6(34):147-149,2013

6. ArvindNarayanan,JosephBonneau,EdwardFelten,AndrewMiller,andSt evenGoldfeder.BitcoinCryptocurrencyTechnologies:AComprehensive Introduction.PrincetonUniversityPress,2016

7. DonTapscott.TheDigitalEconomy:PromiseandPerilintheAgeofNetwor kedIntelligence:McGraw-Hill,1994.

8. Arthur Gervais, Ghassan O. Karame, Karl W ü st,Vasileios Glykantzis, $\mathrm{Hu}$ bertRitzdorf,andSrdja Capkun.On the security and performance of proof of work blockchains. In Proceeding softhe 2016 ACMSIGSAC Conferenceon Computer and Communications Security ,CCS'16,pages3-16,NewYork,NY,USA,2016.ACM.

9. JianjunSun,JiaqiYan,andKemZ.K.Zhang.Blockchain-basedshari ngservices:Whatblockchaintechnologycancontributetosmartcities.Fin ancialInnovation,2(1):26,2016.

10. SarahUnderwood.BlockchainBeyondBitcoin.Commun.ACM,59(11): 15-17,October2016.

11. DannyBradbury.Theproblemwithbitcoin.ComputerFraud\&Security, 20 13(11):5-8,2013.

12. JenniferJ.Xu.Areblockchainsimmunetoallmaliciousattacks?FinancialI nnovation,2(1):25,2016.

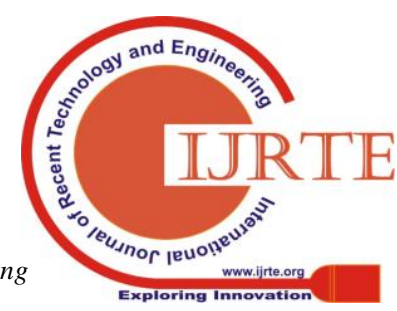


13. NingShi.Anewproof-of-workmechanismforbitcoin.

FinancialInnovation ,2(1):31,2016.

14. Bitcoinwiki; 2015. Accessed: 24/3/2016. https://en.bitcoin.it.

15. J. Garay, A. Kiayias, and N. Leonardos, The Bitcoin Backbone Protocol: Analysis and Applications, pp. 281-310, Springer Berlin Heidelberg, Berlin, Heidelberg, 2015.

16. A. Gervais, G. O. Karame, V. Capkun, and S. Capkun, "Is bitcoin a decentralized currency?,” IEEE Security Privacy, vol. 12, pp. 54-60, May 2014.

17. S. Nakamoto, Bitcoin: A Peer-to-Peer Electronic Cash System, Feb. 24, 2013. (http://bitcoin.org/ bitcoin.pdf)

18. Einstein, A., B. Podolsky, and N. Rosen, 1935, "Can quantum-mechanical description of physical reality be considered complete?", Phys. Rev. 47, 777-

19. YuanfengCaiandDanZhu.Frauddetectionsforonlinebusinesses:aperspe ctive from block chain technology.Financial Innovation,2(1):20,2016

20. J. Van den Hooff, M. F. Kaashoek, N. Zeldovich, "Versum: Verifiable computations over large public logs", Proceedings of the 2014 ACM SIGSAC Conference on Computer and Communications Security, pp. 1304-1316, 2014.

21. J. Bruce, The mini-blockchain scheme, July 2014, [online] Available: http://cryptonite.info/files/mbc-scheme-rev3.pdf.

22. Sean Rowan, Michael Clear, Meriel Huggard and Ciaran Mc Goldrick, "Securing vehicle to vehicle data sharing using blockchain through visible light and acoustic side-channels", eprint arXiv:1704.02553, April,2017.

23. A. Anjum, M. Sporny, A. SillBlockchain standards for compliance and trustIEEE Cloud Comput., 4 (4) (2017), pp. 84-90.

24. M Chung, J Kim, The internet information and technology research directions based on the fourth industrial revolution. KSII Trans. Internet Inf. Syst. 10(3), 1311-1320 (2016).

25. M.S. Adam, M.H. Anisi, I. AliObject tracking sensor networks in smart cities: taxonomy, architecture, applications, research challenges and future directionsFuture Generat. Comput. Syst. (2017).

26. Loi Luu, Duc-Hiep Chu, Hrishi Olickel, Prateek Saxena, and Aquinas Hobor. Making Smart Contracts Smarter. In Proceedings of the 2016 ACM SIGSAC Conference on Computer and Communications Security, CCS ‘16, pages 254-269, New York, NY, USA, 2016. ACM.

27. Al-Hader M, Rodzi A, Sharif AR, Ahmad N (2009) Smart city components architicture, 2009 International Conference on Computational Intelligence, Modelling and Simulation, IEEE., pp 93-97.

\section{AUTHORS PROFILE}

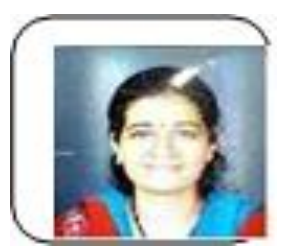

Ammbika V. M Completed Bachelor of Computer Science And Enggineering in 2005 from Shivaji University with First Class and Distinction,Completed MTech in Computer Science and Enggineering from JNTU University with First Class and Distinction, Doctoral Research Scholar in Computer Science And Enggineering from Koneru Lakshmaiah Education Foundation(KLEF),Vaddeswaram,Guntur District Andhra Pradesh-522502,India,,Published papers -1:'Security Challenges with cloud computing" in IJEST ,Volume 4,Issue 10,15

oct.2015,2:"Computer Security with computer protection and network management" in IJESRT volume 5,Issue 6,05 june 2017,3:"Bigdata with Internet Of Things (IOT)" in IJEST volume 06,ISSUE 6,05 june 2017,4:"Balancing Load using parallel. Computing and distributed computing",in IJESRT volume 07,Issue 4,05 April 2018 , membership-Member of Indian Institute of Engineers of India MIE-1538225,Member of ISTE -LM-123599

D.S.Rao, Computer Science and Enggineering,Associate Dean(P \& D), ,Koneeru Lakshmaiah Education Foundation(KLEF) ,Vaddeswaram, Guntur District, Andhra Pradesh-522502, India. 